Comparative Philosophy Volume 10, No. 1 (2019): 127-142

Open Access / ISSN 2151-6014 / www.comparativephilosophy.org

https://doi.org/10.31979/2151-6014(2019).100111

CONSTRUCTIVE-ENGAGEMENT DIALOGUE (1.2)

\title{
PRAGMATIC APPROACH OR CONSTRUCTIVE-ENGAGEMENT STRATEGY?-A METHODOLOGY IN COMPARING CHINESE AND WESTERN PHILOSOPHY
}

\section{WEI SUN}

In the midst of rising interest in cross-cultural comparative philosophy, the methodology of comparisons between Western philosophy and Chinese philosophy have been the focus of scholarship. In Ames' view, as the common philosophical theorization between the two different cultural traditions could hardly be achieved, we could hardly conclude a joined philosophical problem between the two different philosophical traditions. In this case, it is more practical to test whether a solution from one tradition is helpful for solving the problem in another tradition instead of philosophically theorization about the two traditions. In contrast to Ames' pragmatic approach, Mou Bo argues for the constructive-engagement strategy in comparative philosophy. Mou's strategy is to find a common philosophical problem which is jointly concerned by different philosophical traditions. Faced with the common philosophical problems, the different philosophical traditions could have their own answers. In this case, we should integrate and adjust the different answers to find a relatively definite answer for the common philosophical problem. In the case of comparing Xun Zi's Dao and Aristotle's eudemonia, this article argues that we may employ both the pragmatic approach and constructive-engagement strategy. Although there are quite a lot of historical and philosophical differences between Xun $\mathrm{Zi}$ and Aristotle's philosophy, it is in the jointly concerned problem that Xun Zi's philosophy could provide a solution for the disputation of inclusivism and intellectualism in understanding Aristotle.

\section{AMES’ PRAGMATIC APPROACH}

In Thinking through Confucius, Roger T. Ames puts forward the methodology of comparative philosophy. He argues that,

SUN WEI: Associate Professor, Institute of Philosophy, Beijing Academy of Social Sciences, Beijing, China. Email: sunwei1977@gmail.com 
......in the enterprise of comparative philosophy, difference is more interesting than similarity...... we hold that it is precisely this recognition of significant differences that provides an opportunity for mutual enrichment by suggesting alternative responses to problems that resist satisfactory resolution within a single culture. The comparative method employed in this essay has led us to isolate a particular problem within our cultural milieu and then to employ the thought of Confucius as a means of clarifying precisely what is at issue with regard to that problem and to suggest approaches to its reconsideration.......It is transcultural in intent since it seeks to promote that sort of dialogue which eventually may result in a mutual recognition of both commonalities and differences a means of addressing important issues of theoretical and practical concern. (Hall and Ames 1987, 5-6)

For Ames, we should "isolate" a particular problem in one cultural tradition and find the solution to this problem in another cultural tradition. We could see Ames' methodology is emphasizing the importance of finding the solution in one philosophical tradition to a particular problem in another philosophical tradition, though the differences and distinctions between the two philosophical traditions are adequately acknowledged. In this case, the different philosophical traditions could enrich and benefit each other by learning from each other. We could see, in Ames' interpretation, the goal of comparing different philosophical traditions is to find a practical solution to a particular philosophical problem in one tradition, while the differences and similarities in the metaphysical background and the whole philosophical system are laid aside.

In fact, in the comparison, a person will have to employ the language and conception in one's own native cultural tradition to understand and interpret the ideas in another tradition.

The naive assumptions that one can find a neutral place from which to compare different cultural sensibilities or that one can easily take an objective interpretive stance within an alternative culture, while comforting to those compulsively attached to the external trappings of objective scholarship, have led to the most facile and distorted accounts of exoteric thinkers. (Hall and Ames 1987, 12)

Since we have no neutral place from which to compare the different cultural traditions, we must be very careful in the comparison between Chinese and Western philosophies. For this purpose, we must be patient and imaginative in making the valid comparison.

In another book, Anticipating China: Thinking Through the Narratives, Hall and Ames argue that "many of the concepts and doctrines that came to comprise the dominant intellectual inventory of Western culture" constitute "those assumptions which hinder members of Western culture from understanding China on its own terms". (Hall and Ames 1995, xx). Hall and Ames advocate "pragmatism and historicism" that "in the area of comparative studies, philosophical theorizing should be replaced by more concrete, praxix-oriented endeavors". (Hall and Ames 1995, xxi.). In Ames' view, as the philosophical theorization between the two different 
cultural traditions could hardly be achieved, we could hardly conclude a joined philosophical problem both of the traditions concern about. In this case, it is more practical to test or experimentally think (Tan 2016, 158-174) whether a solution from one tradition is helpful for solving the problem in another tradition instead of philosophical theorizing about the two traditions.

In contrast to this pragmatic approach, Mou Bo, however, argues that,

It is noted that the Hall/Ames style pragmatic approach as a whole can be refined into its pragmatic "perspective" dimension and its foregoing pragmatist "guiding-principle dimension; its pragmatic perspective per se can be "eligible" whenever there are such "pragmatic" aspects of objects of study which certain "pragmatic" perspectives are in need to point to and capture; however, an indiscriminate "pragmatist" guiding principle identified above can be "inadequate" because it does not do justice to other "eligible" perspectives but celebrates the pragmatic perspective only. In this sense, and to this extent, the constructive-engagement strategy can do justice to the "pragmatic" methodological perspective while explaining why and in which connections an indiscriminate "pragmatist" methodological guiding principle needs to be regulated. (Mou 2018, 25)

In the next section, we will examine Mou's constructive-engagement strategy in details.

\section{MOU'S CONSTRUCTIVE-ENGAGEMENT STRATEGY}

In the journal of Comparative Philosophy, Mou argues that

One strategic goal and basic methodological strategy of comparative philosophy as understood in a philosophically interesting and significant way can be summarized in this manner: to inquire into how, via reflective criticism (including self-criticism) and argumentation, distinct modes of thinking, methodological approaches, visions, insights, substantial points of view, or conceptual and explanatory resources from different philosophical traditions and/or different styles/orientations of doing philosophy (within one tradition or from different traditions) can learn from each other and jointly contribute to our understanding and treatment of a series of issues, themes or topics of philosophical significance, which can be jointly concerned through appropriate philosophical interpretation and/or from a broader philosophical vantage point. (Mou 2010,3)

That is to say, comparative philosophy focuses on the comparison between different philosophical traditions, in which we could solve some philosophical problem or invent some philosophical ideas. But how could the comparison be valid, considering the various different cultural and philosophical traditions?

Mou argues that,

Although historical descriptions provide necessary sources and data bases for further philosophical interpretation and elaboration, comparative philosophy (as philosophical inquiry) can neither stop at merely giving historical descriptions and being content with 
seeking apparent similarities and differences of thinkers' views or texts under comparative examination without further philosophical interpretation and philosophicalissue-engagement, nor even take the historical-description-giving approach as the exclusively legitimate approach in comparative studies. (Mou 2010, 5)

In this case, we should focus on the philosophical interpretation and engagement in comparative philosophy. In the cross-traditional interpretation, "some effective conceptual and explanatory resources well developed in another tradition or account can be consciously used to enhance our understanding of, and to elaborate, the thinker's ideas/texts." (Mou 2010, 14)

Mou then puts forwards three phases in the comparison:

1. The pre-engagement phase, in which certain ideas from distinct accounts or from different traditions that are relevant to the common concern under examination and thus to the purpose of the project are focused on and identified;

2 . The engagement phase, in which those ideas internally engage with each other in view of that common concern and the purpose to be served; and

3. The post-engagement phase, in which those distinct ideas from different sources are now absorbed or assimilated into a new approach to the common concern under examination. (Mou 2010, 17)

As for the first phase, we should derive some philosophical ideas from the philosophical tradition with a philosophical perspective, while "without involving those irrelevant elements in the account or tradition from which such a perspective comes". The reason is that what we are going to do in comparative philosophy is not studying on the relationship between the idea and its cultural tradition or background, but how the idea is related to the concerned philosophical problem. In the second phase, the philosophical ideas from different cultural traditions are constructively engaged. "From each party's point of view, the other party is something external without; but, from a broader philosophical vantage point and in view of the jointly concerned issue, the distinct views may be complementary within." In the last phase, what we should do is to "adjust, blur and absorb different perspectives into one approach as a whole." (Mou 2010, 18)

As we can see, Ames' approach in comparative philosophy is to find an approach for solving the problem of one philosophical tradition by employing the resources from another philosophical tradition, while setting aside the metaphysical or other philosophical differences between these two philosophical traditions. We can see Ames' approach is rather pragmatic in that it is not going to explore the fundamental metaphysics or ethical similarities or differences between different cultural traditions but just for finding a practical approach for "treating" the "illness" or problem by employing the external "medicine", whether the background and cultural tradition of such "medicine" is how different from us. As long as it is effective, it could be used to solve the problem and improve the understanding of the philosophy in different traditions. 
Mou's constructive-engagement strategy is different from Ames' approach. Mou's strategy is for finding a common philosophical problem which is jointly concerned by different philosophical traditions. While acknowledging the different traditions and cultural background, it focuses on the philosophical problem derived from the different traditions. Faced with the common philosophical problems, the different philosophical traditions could have their own answer. In this case, we should integrate and adjust the different answers to find a relatively definite answer for the common philosophical problem. We can see such strategy is based on the assumption that the different philosophical traditions could have a common metaphysical or ethical foundation, or at least, a common concern in philosophy (such as human nature, justice, etc.), from which we could conclude the common problem in the different traditions.

So, which strategy is more adaptable to the comparative philosophy? Before we can answer this question, let's turn to a comparative examination between Xun Zi's and Aristotle's relevant approaches, from which we might be on a better position to make a valid evaluation.

\section{TOWARD A METHODOLOGY COMBINING AMES' AND MOU'S APPROACHES-A CASE STUDY OF XUN ZI'S DAO AND ARISTOTLE'S EUDAIMONIA}

As for the Dao, Xun Zi said,

The sage knows the flaws of the mind's operation and perceives the misfortunes of blindness and being closed to the truth. This is why he is without desires and aversions, without beginnings and ends of things, without the remote or near, without broadness or shallowness, without antiquity or modernity. He lays out all the myriad things and causes himself to exactly match how each settles on the suspended balance..... What is the balance? I say that it is the Dao. This is why it is inadmissible for the mind not to know the Dao...... Therefore the critical factor necessary to put things in order consists in understanding the Dao. (Knoblock 1994, 21.5a-b.)

Thus, Xun Zi's Dao is a Dao contextualized in "all the myriad things", which dispels personal obsessions. Xun $\mathrm{Zi}$ argued that the teachings of various schools all stick to one point while neglect other schools' reasonable points. Xun Zi said,

Mo Di was blinded by utility and was insensible to the value of good form. Song Xing was blinded by desire and was insensible to satisfaction. Shen Dao was blinded by law and was insensible to worth. Shen Buhai was blinded by technique and was insensible to knowledge. Hui Shi was blinded by propositions and was insensible to realities. Zhuang Zhou was blinded by Nature and was insensible to men. Thus in a doctrine called the Dao grounded on "utility", everyone will be consumed with seeking profit. In a doctrine called the Dao grounded in "desire", everyone will concentrate on seeking satisfaction. In one that grounds everything in "law", every decision becomes wholly a matter of calculation......Each of these methods encompasses but a single corner of the Dao. But 
the Dao itself is constant in its form yet completely changeable. One corner is an insufficient basis for drawing conclusions about it. (Knoblock 1994, 21.4)

We should notice that, in criticizing the teachings of non-Confucian schools, Xun $\mathrm{Zi}$ did not totally reject them but accept the reasonable points in their teachings. For Xun $\mathrm{Zi}$, the reasonable elements in various schools could also be a part of the Dao. However, these non-Confucian teachings could not cover all of Dao. Dao itself includes not only ren (仁 benevolence), yi (義 propriety), $l i$ (禮 ritual) and other Confucian teachings, but also $f a$ (法 law), $c i$ (辭 language and logic), tian (天 nature) and other non-Confucian teachings. Despite the non-Confucian teachings, the Confucian teachings like benevolence and rituals are still the core of Confucian Dao. Xun Zi said,

Only after the gentleman has dwelt with humane principles through justice and morality is he truly humane; only after he conducts himself with justice and morality through ritual principles is he truly just and moral; and only where he regulates with ritual principles, returning to the root and perfecting the branch, is he truly in accord with ritual principles. Only when there three have been made comprehensive has he reached the Dao. (Knoblock 1994, 27.21)

For Xun Zi, Dao is always Confucian Dao, through which ren, yi and $l i$ are linked together. Janghee Lee argues,

Xunzi's fundamental concern seems to lie not in the complete transformation of ordinary xin into achieved xin, but in the articulation of the capacities of xin to allow people to attain the knowledge of $d a o . . . l i$ is closely related to the notion of dao...Li furnishes dao with specific and "formal prescriptions for proper behavior." Accordingly, $l i$ can be deemed one of the core elements that embody the spirit of dao. (Lee 2005, 54-56)

In Lee's view, $l i$ is the core element of Dao, which might include many aspects and dimensions. A. S. Cua also argues,

I have construed tao $[d a o]$ as a generic term that has its fundamental specifications in jen [ren] (benevolence), $l i$ (ritual rules), and $i$ [yi] (rightness). Contrary to this interpretation, it is commonly held that $l i$ is Hsun Tzu [Xun Zi]'s basic concept. It cannot be denied that $l i$ occupies a central place in Hsun Tzu's philosophy. But if we follow this interpretation, we need to regard $l i$ as an inclusive virtue that embraces both $j e n$ and $i$, and other virture. (Cua 1985, 160-161)

Thus, in Cua's view, it is Dao rather than $l i$ that dominates Xun Zi's philosophies and Confucian teachings. I wish to push his point further: Xun Zi's Dao is a much broader concept that may include not only the Confucian doctrines such as $l i$, ren, yi, etc., but also some useful doctrines from other schools, including $f a$, which could be utilized to develop Confucianism to be more comprehensive. 
Happiness is the English translation of Greek word eudaimonia. In its origin, eudaimonia has the implication of "loving for the God" and is related with human flourishing or good luck. From Socrates, eudaimonia bears the characteristic of "doing well" or "living well". For the ancient Greeks, eudaimonia is the ultimate goal for the human life. Aristotle's eudaimonia also followed such ancient Greek tradition and explicated its meanings in a more explicit way.

To understand Aristotle's eudaimonia, we will have to examine the core element in this concept, that is, the good. Aristotle said,

Even if there is some good which is universally predicable of goods or is capable of separate and independent existence, clearly it could not be achieved or attained by man; but we are now seeking something attainable......For all of these sciences, though they aim at some good and seek to supply the deficiency of it, leave on one side the knowledge of the good. (1097a5-7)

Then, does it mean that the ultimate goal of human life could be attained when they attained the level of virtue? For this question, Aristotle would say no. For him, virtue is not the ultimate goal of human life. Instead, it is eudaimonia rather than other virtues is the ultimate goal people should pursue. Eudaimonia is not equivalent to virtue, which is only a state of character (1106a11-12). Although the argument of virtue occupies a central place in Aristotle's ethics, the purpose of virtue and Aristotle's ethics is for attaining eudaimonia. In the process of realizing the eudaimonia, virtue is only the first step. Aristotle said,

With those who identify happiness with virtue or some one virtue our account is in harmony; for to virtue belongs virtuous activity. But it makes, perhaps, no small difference whether we place the chief good in possession or in use, in state of mind or in activity. For the state of mind may exist without producing any good result, as in a man who is asleep or in some other way quite inactive, but the activity cannot; for one who has the activity will of necessity be acting, and acting well. (1098b30-1099a3)

We could see, Aristotle's happiness is not the possession of the virtue, which is only a state of character, but the use of virtue in practice, that is, the virtuous activity. Aristotle said,

Since, then, the present inquiry does not aim at theoretical knowledge like the others (for we are inquiring not in order to know what virtue is, but in order to become good, since otherwise our inquiry would have been of no use), we must examine the nature of actions, namely how we ought to do them; for these determine also the nature of the states of character that are produced, as we have said. (1103b27-31)

We have known that a person should put his virtue into practice so as to achieve the happiness. However, asides from such virtuous practice, we also need other conditions to attain the level of happiness. In Aristotle's words, such conditions are "external goods". Aristotle said, 
It needs the external goods as well; for it is impossible, or not easy, to do noble acts without the proper equipment. In many actions we use friends and riches and political power as instruments; and there are some things the lack of which takes the luster from happiness, as good birth, goodly children, beauty; for the man who is very ugly in appearance or ill-born or solitary and childless is not very likely to be happy...... As we said, then, happiness seems to need this sort of prosperity in addition; for which reason some identify happiness with good fortune, though others identify it with excellence. (1099a31-1099b7)

Thus, the external goods such as good birth, good children and beauty are the necessary conditions for attaining happiness. However, the external goods are not determined by someone's willingness, instead, it is determined by the luck (1099b15). A person lives virtuously with the accompany of external goods could be regarded as happiness.

All of the above definitions and descriptions of happiness are what Aristotle argued in the first nine chapters of Nicomachean Ethics. To conclude all of these definitions, we could see that happiness is an integrated body which includes both virtuous activities and external goods. However, in Chapter 10 of Nicomachean Ethics, which is also the last chapter, Aristotle mentioned the features of happiness again. He concluded that,

We said, then, that it (happiness) is not a state; for if it were it might belong to some one who was asleep through his life, living the life of a plant, or, again, to some one who was suffering the greatest misfortunes. If these implications are unacceptable, and we must rather class happiness as an activity, as we have said before, and if some activities are necessary, and desirable for the sake of something else, while others are so in themselves, evidently happiness must be placed among those desirable in themselves, not among those desirable for the sake of something else; for happiness does not lack anything, but is self-sufficient. Now those activities are desirable in themselves from which nothing is sought beyond the activity. And of this nature virtuous actions are thought to be; for to do noble and good deeds is a thing desirable for its own sake. (1176a33-b9)

Thus, we will find that the statement "happiness does not lack anything, but is selfsufficient" seems contrary to the former argument of happiness. In the former nine chapters, happiness, which consists of virtuous activities and external goods, is not so sufficient as what is defined in chapter 10. In fact, Aristotle regarded virtue itself as limited and insufficient. Aristotle said,

The self-sufficiency that is spoken of must belong most to the contemplative $(\theta \varepsilon \omega \rho \imath \alpha)$ activity...... And this activity alone would seem to be loved for its own sake; for nothing arises from it apart from the contemplating, while from practical activities we gain more or less apart from the action. (1177a28-1177b4) 
In this sense, virtuous activities, from which we gain more or less, are not sufficient. However, contemplative activities are not dependent upon anything and so it is totally sufficient by itself.

There are quite a lot of debates over eudaimonia in the scholarship. The debate normally focuses on the understanding of eudaimonia as virtuous activities and external goods or the contemplative activities. Some scholars argue that the happiness includes both virtuous activities and external goods, which could be labeled as "inclusivism". (Cooper 1999) Some scholars argue that happiness refers only the contemplative activities, which could be labeled as "intellectualism". (Reeve 1992) Other scholars argue that Aristotle swings between "inclusivism" and “intellectualism". (Broadie 1991)

Thus, how to understand eudaimonia? It refers to the virtuous activities or just intellectual (contemplative) activities? To answer this question, we will have to examine the concept of contemplation $(\theta \varepsilon \omega \rho \imath \alpha)$ at first.

In Metaphysics, Aristotle argued,

Therefore it (nous) must be of itself that the divine thought thinks (since it is the most excellent of things), and its thinking is a thinking on thinking. (1074b33-5)

This means that nous is same as what it thinks, which is itself. Aristotle said further,

We answer that in some cases the knowledge is the object. In the productive sciences, it is the substance or essence of the object, matter omitted, and in the theoretical sciences the definition or the act of thinking is the object. Since, then, thought and the object of thought are not different in the case of things that have not matter, the divine thought and its object will be the same, i.e. the thinking will be one with the object of its thought. (1074b38-1075a5)

This is to say, the thinking should be the thinking of what the most excellent in oneself. For the God, thinking is thinking of himself; while for the human beings, thinking is thinking of what is best in himself, that is, the rationality. Here, contemplation $(\theta \varepsilon \omega \rho \iota \alpha)$ is the speculation of things that have not material substance (including philosophy, God or nous).

For Aristotle, such contemplation could help a person to attain the ultimate happiness. In chapter 7 of Nicomachean Ethics X, Aristotle stated some characteristics of contemplation:

First, contemplation is the best and, secondly it is the most continuous, since we can contemplate truth more continuously than we do anything. And we think happiness has pleasure mingled with it, but the activity of philosophic wisdom is admittedly the pleasantest of virtuous activities...... And the self-sufficiency that is spoken of must belong most to the contemplative activity. (1177a20-29)

Here, we could compare the features of contemplation with those of happiness to see whether contemplation could satisfy all the conditions of happiness. 
First, contemplation is an activity, rather than a disposition. Contemplation is an intellectual activity. Second, contemplation is a sufficient activity. Third, contemplation is chosen by itself rather than by other purpose. Fourth, contemplation is an activity in accordance with the proper excellence. Aristotle said,

If happiness is activity in accordance with excellence, it is reasonable that it should be in accordance with the highest excellence; and this will be that of the best thing in us......the activity of this in accordance with its proper excellence will be complete happiness. That this activity is contemplative we have already said. (1177a7-18)

From the above four descriptions, we will find that the contemplation satisfies all the conditions of happiness. In this sense, we could say that contemplation is the core of the happiness.

Although the contemplation is the core of happiness, we might not say that virtuous activities that Aristotle related to happiness are not happiness. In fact, despite the insufficiency, the virtuous activities could be regarded as the "secondary happiness" while the contemplation is the "primary happiness". So, how could the pure theoretical contemplation be compatible with the virtuous activities? Before we answer this question, let us see what Xun $\mathrm{Zi}$ said about the similar question.

In Xun Zi's view, Confucian Dao is an integrated body that consists of various virtuous activities and external goods. However, we might easily neglect an important concept here, that is, “da-qing-ming” (大清明 Great Pure Understanding). So, what is da-qing-ming? How is it related to the virtuous activities? Xun Zi said,

A person who knows the Dao and discerns it and puts it into practice embodies the Dao. Emptiness, unity, and stillness are called the Great Pure Understanding (da-qing-ming). Each of the myriad things has a form that is perceptible. Each being perceived can be assigned its proper place. Each having been assigned its proper place will not lose its proper position. Although a person sits in his own house, yet he can perceive all within the four seas. Although he lives in the present, he can put in its proper place what is remote in space and distant in time...... Extensive and complete, broad and wide-who knows his limits? Bright and luminous, brilliant and shining-who knows his inner power? Rolling and bubbling, multitudinous and multifarious-who can know his external form? Brightness comparable to the sun and moon; greatness filling the Eight Poles - such a person is truly what is meant by "Great Man." How indeed could he have obsessions!(Knoblock 1994, 21.5d 21.5e)

From the above quoted, we could see, the Great Pure Understanding is the status when we attain the Emptiness, unity and stillness. So, what is "emptiness, unity and stillness"? Xun Zi said,

What do men [humans] use to know the Way [Dao]? I say that it is in the xin. How does the xin know? I say by its emptiness, unity, and stillness. The xin never stops storing; nonetheless it possesses what is called emptiness. The xin never lacks duality; 
nonetheless it possesses what is called unity. The xin never stops moving; nonetheless it possesses what is called stillness. (Knoblock 1994, 21.5d)

Xun $\mathrm{Zi}$ argued that we should accommodate the myriad things and various views to avoid prejudices, but how could a person accomplish this? Xun Zi said,

Men [humans] (Ren 人) from birth have awareness. Having awareness, there is memory. Memories are stored in the mind, yet the mind has the property called emptiness. Not allowing what has previously been stored to interfere with what is being received in the mind is called emptiness. (Knoblock 1994, 21.5d) ${ }^{1}$

Although we have stored in our xin (心) memories of the knowledge we have perceived, xin should not be totally preoccupied with such previous knowledge to the point of neglecting the importance of future learning. Even if what we are going to learn is different from or contradicts what we have learnt, we should be able to entertain it without prejudice and understand it on its own terms. That is to say, regardless of how much knowledge we have stored in our xin, we should reserve enough room for accommodating new knowledge. Only in this way could the previous knowledge serve as the basis of our future learning. Also, only in this way would future learning add to our knowledge persistently. Thus, Xun Zi employed the method of $x u$ (虛) to attain the detached mind to accommodate various views in the xin.

We should notice that Xun Zi's "emptiness" is not total emptiness. In other words, the previous knowledge should still be stored in the xin so that the further acquired knowledge could be gained in addition to previous knowledge. Xun Zi said, "The xin never stops storing..." (Knoblock 1994, 21.5d) For Xun Zi, the usage of $x u$ is for cultivating xin into an ideal state so as to know the Way.

What do men use to know the Way? I say that it is the mind. How does the mind know? I say by its $x u$ (emptiness), unity, and stillness. (Knoblock 1994, 21.5d)

So, for Xun $\mathrm{Zi}$, the usage of $x u$ is for dispelling prejudices in the $x$ in and knowing the Confucian way comprehensively. This is a moral epistemological purpose within the context of personal cultivation and good government.

For Xun Zi, to establish the Confucian way and adapt what is learnt to the current circumstance, a person must open his mind and broaden it to avoid prejudices and accommodate more views. But how could such diverse views adopted from various schools be kept coherent and formulated into a set of systematic knowledge? This requires $y i$ (喜 unity).

\footnotetext{
${ }^{1}$ As some commentators (Tao Hongqing 陶鴻慶 and Wang Tianhai 王天海) observe, ren (humans) here should be xin (heart/mind), considering the meaning of following paragraphs. See Wang 2005, 849-850.
} 
The xin from birth has awareness. Having awareness, there is perception of difference. Perception of difference consists in awareness of two aspects of things at the same time. Awareness of two aspects of things all at the same time entails duality; nonetheless the xin has the quality called $y i$. Not allowing the one thing to interfere with the other is called yi. (Knoblock 1994, 21.5d)

$Y i$ in the Xun-Zi refers to xin's function of maintaining coherence in knowing. This means: although we may have perceptions of different aspects among various things or in a thing simultaneously, xin could concentrate on one aspect while at the same time keeping all the other aspects coherent. The xin would not let the perception of other aspects interrupt the perception of the current aspect, while these different perceptions could be kept coherent by xin's function of $y i$.

For Xun $\mathrm{Zi}$, since humans should govern and utilize the myriad things in the world to serve themselves, they should first try to know the myriad things. People could have different views of the world and this is the reason why the different schools exist in the world. But since the myriad things are unified, the knowledge of them should also be unified instead of being separated. In this sense, the different schools' views, which could be regarded as different aspects of the whole truth, should be combined and unified into a whole so as to realize the final truth. That is to say, the various views of different schools should be unified to formulate a holistic view of the world. Xun Zi said,

The myriad things constitute one aspect of the Way, and a single thing constitutes one aspect of the myriad things. The stupid who act on the basis of one aspect of one thing, considering that therein they know the Way, are ignorant. (Knoblock 1994, 17.12)

For Xun Zi, since all views should be unified, we should not only focus on one particular view but should keep the different views of all things unified and coherent. Only in this way, we might be able to know the world comprehensively. For Xun Zi, emptying xin and accepting the different views is only the first step for knowing the truth. The next step is to unify these different perceptions and fit them together so as to achieve a full and good understanding.

After $x u$ and $y i$, it seems that a person could have formulated the most comprehensive knowledge of the world. However, for $\mathrm{Xun} \mathrm{Zi}$, there is another necessary condition for achieving this goal which is jing (靜 stillness) in the xin. Without jing, $x u$ and $y i$ could not be completed smoothly. Xun Zi said,

When the mind is asleep, it dreams. When it relaxes, it moves of its own accord. When it is employed in a task, it plans. Thus the mind never stops moving; nonetheless it possesses the quality called stillness. Not allowing dreams and fantasies to bring disorder to awareness is called stillness. (Knoblock 1994, 21.5d.)

"Jing" in the Xun Zi means that we should overcome all kinds of illusions or dreams in the xin so as to realize the Way. Since we all have illusions or imaginations in our everyday life, such as dreams, nonsense thoughts, illusions, etc., we should not let 
such illusions distract our thinking. For Xun Zi, xin should be kept still just like the still water to reflect the Way without distortion. Xun Zi said,

The human mind may be compared to a pan of water. If you place the pan upright and do not stir water up, the mud will sink to the bottom, and the water on top will be clear and pure enough to see your beard and eyebrows and to examine the lines on your face. But if a slight wind passes over its surface, the submerged mud will be stirred up from the bottom, and the clarity and purity of the water at the top will be disturbed so that it is impossible to obtain the correct impression of even the general outline of the face. Now, the mind is just the same. (Knoblock 1994, 21.7b)

Thus, after attaining the status of “ $x u, y i$ and jing” (虚壹而静), that is, the status of "da-qing-ming", a person could have a most comprehensive mind to accommodate the various views of the world so as to attain the Dao. Here, we could see that Xun Zi's "da-qing-ming" is beyond virtuous activities. "Extensive and complete, broad and wide - who knows his limits? Bright and luminous, brilliant and shining-who knows his inner power? Rolling and bubbling, multitudinous and multifarious-who can know his external form?" seems to say that, virtuous activities have some limits and concrete forms, while "da-qing-ming" is beyond the external limits and is sufficient by itself. Although it has no concrete forms, it embodies various virtuous activities and even the fundamental principles of the cosmos. Once a person attains the status of "da-qing-ming", he realizes the Dao. In this aspect, we could see that Xun Zi's way of attaining "da-qing-ming"_- $x u, y i$ and jing" — is quite close to Aristotle's " $\theta \varepsilon \omega \rho \imath \alpha$ " (contemplation).

Xun Zi's " $x u$, yi and jing" and Aristotle's " $\theta \varepsilon \omega \rho l \alpha "$ (contemplation) have many similarities, in which Aristotle's " $\theta \varepsilon \omega \rho \iota \alpha$ " is for achieving the highest happiness, while Xun Zi's " $x u$, yi and jing" is for attaining the highest dao. Happiness and Dao are the ultimate purpose and status for the two philosophers. In this case, could we say that happiness and dao only lies in " $\theta \varepsilon \omega \rho \imath \alpha$ " and " $x u$, yi and jing"? It seems too rash to do so. For Aristotle, if happiness only lies in " $\theta \varepsilon \omega \rho \imath \alpha$ ", he seems not necessary to talk about happiness in terms of virtuous activities and external goods in the former nine chapters of Nicomachean Ethics. For Xun Zi, if dao lies only in " $x u$, $y i$ and jing", then he seems not necessary to talk about virtuous practices and ritual principles in the rest chapters of $X u n-Z i$. It seems to indicate that, " $\theta \varepsilon \omega \rho \imath \alpha$ " and " $x u$, $y i$ and jing" are not the full of happiness or Dao, although they are the cores of them.

In Xun Zi's view, although a person could attain the Dao when he achieves the status of "da-qing-ming", this highest status does not dispel the Dao at the lower level. Virtuous activities and ritual principles are the expressions of "da-qing-ming" in the realistic world, which belongs to a lower-level Dao. All of these are the elements of the Dao. That is to say, it is right to say that Confucian Dao is the combination of virtuous activities and external goods. However, we must notice that a higher-level Confucian Dao is the reflection of the virtuous activities or even the cosmos orders. Aristotle's " $\theta \varepsilon \omega \rho \imath \alpha$ " is quite similar to Xun Zi's " $x u$, yi and jing". 
" $\theta \varepsilon \omega \rho \imath \alpha "$ is also the theoretical reflection of the human rationality and cosmos nous, which is not limited to some specific virtuous activity.

In this aspect, Xun Zi's view could provide a solution for the disputation between inclusivism and intellectualism among Aristotle's research. That is to say, when we make clear the position of " $x u$, yi and jing" and " $\theta \varepsilon \omega \rho 1 \alpha$ " in Xun Zi and Aristotle's philosophies and their relationship with virtuous activities and external goods, we could stand on a better position to explain such a problem, that is, why Aristotle argued that virtuous activities and external goods are happiness in the former nine chapters of Nicomachean Ethics, while arguing that contemplation is the highest happiness at the end of the book? Or we may ask, if Nicomachean Ethics is a consistent book, how the highest happiness be compatible with virtuous activities and external goods that are concluded as the low-level happiness?

We know that, Xun $\mathrm{Zi}$ argued that the realization of Dao lies in the attainment of "da-qing-ming", which is realized by the activities of " $x u, y i$ and jing". After the activities of " $x u, y i$ and jing", we could accommodate more views of cosmos and human life. Various virtuous activities and moral practices is the foundation of " $x u, y i$ and jing", which does not dispel the virtuous activities. In this case, "da-qing-ming" and moral practices are compatible as the former is the self-reflection of the latter and the latter is the expression of the former in practice.

The status of "da-qing-ming" includes the reflection of virtuous activities and external goods, so it must be the highest level of Dao. If we can compare Xun Zi's " $x u$, yi and jing" to Aristotle's " $\theta \varepsilon \omega \rho \imath \alpha "$, we could say that " $\theta \varepsilon \omega \rho \imath \alpha$ " is also the theoretical reflection of virtuous activities and external goods, so it must be the highest level of eudaimonia. Thus, if we only say on the highest level, Aristotle's eudaimonia is contemplation, which is also the intellectualism argued for.

On the other hand, if we say it on a lower level, Xun Zi's Dao is virtuous activities and external goods and Aristotle's happiness is the combination of virtuous activities and external goods, which is not the highest level of happiness and could not render the essence of happiness.

That is to say, if we treat the virtuous activities and external goods as happiness, this is not wrong and we could render it as "practical happiness". But we should also notice that, if we call contemplation as happiness, this is also not wrong, because contemplation includes the features of the perfect virtuous activities and is selfsufficient. In this case, we could render it as "theoretical happiness". In fact, only the virtuous activities are promoted to the theoretical level, it could become the highest happiness because the virtues are quite limited to the specific circumstance and situation, while contemplation is beyond all circumstances and situation so that it is the highest happiness. Thus, if we treat Aristotle's happiness as a consistent system, we could not treat the two understandings of happiness so extremely. In fact, practical happiness and theoretical happiness should be combined together. As Richard Kraut pointed out,

Aristotle thinks that the most eudaimon individual is someone who has fully developed and regularly exercises the various virtues of the soul, both intellectual and moral. Such a 
person engages in philosophical activity (since this is the full flowering of his capacity to reason theoretically) and also in moral activities, which display his justice, generosity, temperance, etc. (Kraut 1979, 170)

The essence of Dao and eudaimonia lies in " $x u$, yi and jing"and " $\theta \varepsilon \omega \rho 1 \alpha$ ", while in the reality, it is always rendered as the virtuous activities and external ritual or political principles. Dao and eudaimonia not only exist in the divine contemplation, but also in the practical virtuous activities and political systems.

As we can see, in the comparison between Xun $\mathrm{Zi}$ and Aristotle's philosophy, we may employ both the pragmatic approach and constructive-engagement strategy. First, we could identify a jointly concerned philosophical problem in Xun $\mathrm{Zi}$ and Aristotle, that is, Dao and eudemonia, and " $x u$, yi and jing" and " $\theta \varepsilon \omega \rho ı$ ". Under such assumption, we could employ the understandings of Dao to solve the problem in the understandings of eudemonia. In fact, a seemingly trivial problem in one philosophical tradition may be tracked to its metaphysical foundation and ethical explication. In this case, we will have to find the common metaphysical or ethical foundations among the objects of comparison, from which we might make the valid engaging comparison. As Mou Bo points out,

Are there any fundamentally shared common bases which serve as norms on which the debating parties (need to) observe or agree on and which can serve as one criterion (if not the exclusive criterion) by which to make due evaluative judgment in this connection? Indeed, if otherwise..., it can be hardly really engaging: the debating parties then might either talk about different objects (instead of talking about the same object differently) and thus essentially pass by each other or have no basis on which to understand and evaluate each other in some substantial connections. (Mou 2019, 187)

In this case, we might not neglect the philosophical theorization in the comparative philosophy. After finding the jointly concerned problem, we can find the practical solutions to the problem. In terms of the understanding of Xun Zi's Dao, we could find that the way for attaining Dao is virtuous activities, which could be compared to the virtuous activities in Aristotle. In the next step, we find that the crucial phase in attaining Dao is " $x u, y i$ and jing", which is quite similar to the process of " $\theta \varepsilon \omega \rho \imath \alpha$ " in Aristotle. In this sense, we could understand the relationship among the virtuous activities, " $\theta \varepsilon \omega \rho \imath \alpha$ " and eudemonia by employing the understandings of Xun Zi's virtuous activities, " $x u$, yi and jing” and Dao. Although there are quite a lot of historical and philosophical differences between Xun Zi's and Aristotle's philosophies, it is in the jointly concerned problem that Xun Zi's philosophy could provide a solution for the disputation of inclusivism and intellectualism in understanding Aristotle. 


\section{ACKNOWLEDGEMENTS}

The early draft of this article is presented on the invited session "Doing Philosophy Comparatively: How to look at distinct approaches in philosophy and society" at the 24th World Congress of Philosophy in Beijing, 2018. I am grateful for the critical comments from the various conference attendants and especially grateful for Prof. Mou Bo's kind help in providing valuable materials in writing this article. I am also grateful for the financial support from Beijing Academy of Social Sciences.

\section{REFERENCES}

Ames, Roger T. and David L. Hall (1987), Thinking through Confucius (Albany: State University of New York Press).

- (1995), Anticipating China: Thinking Through the Narratives of Chinese and Western Culture (Albany: State University of New York Press).

Aristotle, Nicomachean Ethics, Jonathan Barnes (ed.) (1984), The Complete Works of Aristotle, vol.2 (Princeton: Princeton University Press).

Broadie, Sarah (1991). Ethics with Aristotle (New York: Oxford University Press).

Cooper, John M. (1999), Reason and Emotion (Princeton: Princeton University Press).

Cua, A. S. (1985), Ethical Argumentation: A Study in Hsün Tzu's Moral Epistemology (Honolulu: University of Hawaii Press).

Knoblock, John (1994), (trans.) Xun Zi-A Translation and Study of the Complete Works, vol.3 (Stanford: Stanford University Press).

Kraut, Richard (1979), "Two Conceptions of Happiness", The Philosophical Review 88.2:170.

Lee, Janghee (2005), Xun Zi and Early Chinese Naturalism (New York: State University of New York Press).

Mou, Bo (2010), "On Constructive-Engagement Strategy of Comparative Philosophy: A Journal of Theme Introduction", Comparative Philosophy 1.1: 1-32.

- (2018), "General Introduction: Constructive-Engagement Strategy to Explore Chinese Philosophy", in Bo Mou (ed.) (2018), Chinese Philosophy: Critical Concepts in Philosophy (London: Routledge), 1-28.

- (2019), Semantic-Truth Approaches in Chinese Philosophy: A Unifying Pluralist Account (Lexington Books).

Reeve, C. D. C. (1992), Practices of Reason (Oxford: Clarendon Press).

Tan, Sor-hoon (2016), "Confucianism and Pragmatist Methods: Keeping Faith with the Confucian Moral Mission", The Bloomsbury Research Handbook of Chinese Philosophy Methodologies (London: Bloomsbury Academic Publishing).

Wang Tianhai (2005), Xun-Zi-Jiao-Shi《苟子校釋》[Annotations on Xun-Zi] (Shanghai: Shanghai-Gu-Ji-Chu-Ban-She). 